\title{
El proceso de toma de decisiones para la tercerización de funciones logísticas: prácticas mexicanas versus mejores prácticas establecidas
}

\author{
María del Pilar Ester Arroyo López* \\ Juan Gaytán Iniestra** \\ Selene Sierra Vilchis***
}

\section{Resumen}

La tercerización de funciones logísticas es una práctica que exhibe una tendencia creciente debido a que se reconoce como opción para decrecer costos, incrementar la eficiencia de las operaciones logísticas y lograr una cadena de suministros más flexible e integrada; sin embargo la pérdida del control directo de las actividades y la incapacidad de los proveedores de servicios logísticos para dar un servicio de excelencia son problemas potenciales cuando se terceriza; por ello es importante hacer una cuidadosa selección de las terceras partes que darán el servicio, así como establecer la planeación para lograr establecer una relación efectiva.

A través del análisis de casos de estudio, este trabajo describe el proceso de toma de decisiones que siguen empresas ubicadas en México cuando tercerizan funciones logísticas; los casos se analizan siguiendo el modelo normativo de Sink y Langly (1997); asimismo, se identifican aquellas prácticas que se desvían de las recomendaciones establecidas y se cuestiona su efectividad, para lo cual se proponen acciones y métodos para la toma de decisiones en las varias etapas del proceso que vayan de acuerdo con las políticas y condiciones del ambiente mexicano.

Palabras clave: cadena de abastecimiento, tercerización en logística, relaciones de asociación en logística, toma de decisiones.

* Profesora de la Escuela de Negocios y Humanidades del Instituto Tecnológico y de Estudios Superiores de Monterrey (ITESM), campus Toluca. Correo electrónico: pilar.arroyo@itesm.mx

** Programa de Doctorado en Ingeniería Industrial del Instituto Tecnológico y de Estudios Superiores de Monterrey (ITESM), campus Toluca. Correo electrónico: jgaytan@itesm.mx

***Profesora de la Escuela de Negocios y Humanidades del Instituto Tecnológico y de Estudios Superiores de Monterrey (ITESM), campus Toluca. Correo electrónico: selene_sierra@hotmail.com 


\section{Introducción}

— $\mathrm{n}$ la búsqueda de una cadena de abastecimiento más eficiente y flexible, las ¿empresas han recurrido a la contratación de terceros para la fabricación de productos, y más recientemente para la realización de servicios. La subcontratación de actividades de logística hacia y desde la empresa (inbound and outbound logistics) es una práctica que ha crecido en las empresas a lo largo de los últimos años, la cual se asocia con las siguientes ventajas: mejoras en el servicio a cliente, simplificación de los procesos logísticos, eficiencia en la realización de actividades logísticas, reducción en la inversión en infraestructura de apoyo y la concentración en actividades para las cuales la empresa está mejor calificada (Bradyley, 1995). Entre los servicios que se confieren a terceras partes (de ahora en adelante se denominará "tercerización" a la práctica administrativa que en inglés se denomina outsourcing) están como candidatos iniciales las funciones logísticas de soporte tales como transporte, administración de almacén y manejo de materiales (Lieb y Randall, 1996); sin embargo, a medida que el ambiente de negocios exige mayor competitividad de las empresas, funciones estratégicas como los sistemas de información logísticos son también tercerizadas (Insinga y Werle, 2000), para lo cual se hace necesario que la relación con el proveedor vaya más allá de establecer un contrato básico que estipule las condiciones del servicio.

Daugherty y Pittman (1995) discurren que la contratación de terceras partes que proveen servicios logísticos (identificados como 3PL por sus siglas en inglés) contribuye no sólo a que la empresa adquiera servicios especializados sin tener que invertir en ellos, sino que además contribuye a crear valor para los consumidores a través de un servicio diferenciado. Este valor agregado se incrementa a medida que las 3PL's extienden el alcance de sus prestaciones al agregar servicios suplementarios a una actividad logística básica y al expandir las alianzas horizontales de la cadena de suministros (Van Hoek, 2001); por ejemplo, los servicios de una 3PL pueden incluir ensamble final, empaque y administración del inventario, como servicios complementarios a las actividades básicas de transporte o almacenaje. A medida que el rango de servicios que realiza la 3PL se amplía, o cuando la actividad logística que se terceriza deja de ser rutinaria y de soporte (más crítica) se incrementa la dependencia de la empresa hacia el proveedor, lo que incrementa el riesgo asociado a una mala decisión en la selección de la tercera parte. 
El proceso de toma de decisiones para la tercerización de funciones logísticas:

prácticas mexicanas versus mejores prácticas establecidas

Lonsdale (1996) argumenta que los fracasos en la tercerización de cierta actividad logística pueden reducirse si quienes toman las decisiones consideran la naturaleza de las actividades que van a ser tercerizadas (críticas o fuente de ventaja competitiva versus no críticas); si analizan la estructura del mercado de proveedores para la función logística (número de proveedores, confiabilidad y capacidad) y las implicaciones asociadas con la transferencia del control de una gama de actividades del negocio. La empresa debe, por tanto, considerar no sólo el precio que pagará por la contratación del servicio o las capacidades técnicas de las 3PL's, sino todo un conjunto de características deseables para un proveedor logístico, que le permitan a través de la tercerización combinar las ventajas competitivas de la empresa con las fortalezas de los operadores logísticos. Desde esta perspectiva, además de los criterios técnicos y de producción, criterios comerciales y sociales tales como la habilidad del proveedor para resolver problemas conjuntamente, la compatibilidad cultural y organizacional entre proveedor y empresa, así como la capacidad para desarrollar una relación de confianza y de largo plazo, también son consideraciones (muy) importantes, además de los criterios básicos de costos y capacidad de servicio (Boyson, Corsi y Rabinovich, 1999).

Varios autores han sugerido modelos que van desde los conceptuales hasta los prescriptivos con el objetivo de comprender y guiar el complejo proceso de las compras organizacionales bajo el contexto actual de negocios, en el cual las empresas han incrementado y globalizado fuertemente sus actividades de compra de productos y servicios (Webster y Wind, 1972; Anderson et al., 1987). Si bien una relación de tercerización logística implica la compra de un servicio, la definición de Africk y Calkins (1994) distingue este tipo de relación en la compra de servicios básicos, indicando que corresponde a una oferta tropicalizada a las necesidades del cliente, en la que se involucra un amplio número de actividades con un enfoque de largo plazo y en la que se persiguen beneficios mutuos. Estas cualidades de la relación de tercerización - largo plazo, enfoque a necesidades del cliente, simetría y complejidad en la relación - han llevado a analizar las relaciones de tercerización desde las perspectivas de relaciones de mercadotecnia, de interacciones organizacionales y personales, además de formación de alianzas estratégicas, para ello se proponen modelos basados en el análisis del proceso de decisión. Estos modelos reconocen cuatro etapas fundamentales que requieren de ser racionalizadas: 1) el reconocimiento de la necesidad de tercerizar, 2) la búsqueda y selección de proveedores, 3) la instrumentación de la relación, y 4) el control y evaluación de la relación. Algunos de los modelos desarrollados en la 
literatura tratan de establecer recomendaciones prácticas para las cuatro etapas del proceso a través de la identificación de actividades, decisiones y métodos apropiados para completarlas, en tanto que otros tienden a enfatizar la relevancia de alguna etapa y las consecuencias de las decisiones tomadas. A continuación, se describen cuatro de los modelos reportados en la literatura y que constituyen el marco de referencia identificado para contrastar las prácticas de tercerización nacionales.

El modelo propuesto por Lonsdale (1996) se centra en evaluar la conveniencia de entablar una relación de tercerización para una actividad de negocios genérica. El proceso de tercerización inicia con la clasificación de la actividad como fundamental (core) o no-fundamental. En caso de que la actividad sea no-fundamental, se considera como buena opción tercerizarla porque el riesgo de hacerlo es bajo; mientras que si la actividad es medular (core), lo que corresponde a una tercerización estratégica se prescribe analizar con detalle la estructura del mercado de proveedores; en cambio si el mercado es competitivo y los costos de cambiar a otra alternativa no son en extremo altos, la recomendación es tercerizar; ahora bien, cuando el mercado tiene pocos proveedores o sólo unos cuantos son competitivos técnicamente, se propone reconsiderar la tercerización y optar por las alternativas de: a) desarrollo de proveedores o b) no transferir la actividad, pero buscar cómo mejorar su eficiencia.

Por otra parte, el modelo propuesto por McIvor (2000) parte también de deliberar sobre la conveniencia de tercerizar, pero considera además la etapa de selección y evaluación de proveedores. En esta etapa, se hace énfasis en que se evalúe a los proveedores no sólo bajo los términos de sus capacidades técnicas y el costo de su servicio, sino también de su potencial para establecer una relación de asociación con el comprador. Lo anterior implica evolucionar de una relación transaccional hacia una relación con visión de largo plazo, basada en la confianza y en la cual ambas partes comparten riesgos, inversiones y trabajan en conjunto para dar solución a los problemas. McIvor coincide con Londsdale en cuanto a la tercerización estratégica, y propone conducir un análisis de costos tipo ABC y un benchmarking del mercado de proveedores para establecer la viabilidad de la decisión, o bien considerar como alternativa el desarrollo de proveedores internos.

Mientras que estos autores establecen trascendentales recomendaciones para las primeras dos etapas del proceso de tercerización, al hacer notar la necesidad de conocer el mercado de proveedores logísticos y el uso de múltiples criterios para elegir a un proveedor (técnicos y de relación), no consideran las decisiones y proce- 
dimientos requeridos en subsecuentes etapas del proceso. Entre los modelos propuestos en la literatura que consideran etapas subsecuentes del proceso destacan el de Sink y Langley (1997) y el de Bagchi y Virum (1998). Estos modelos sugieren acciones derivadas de las prácticas empresariales observadas; además, reconocen que el proceso de tercerización es iterativo, lo que hace necesario retornar a etapas anteriores para revisar las decisiones. La propuesta de Sink y Langley (SL) corresponde a un modelo normativo, el cual se basa en información de cuatro fuentes: a) información bibliográfica de diversas áreas del conocimiento relacionadas con logística, b) grupos de enfoque con ejecutivos de logística, c) una encuesta (a juicio) aplicada a ejecutivos de alto nivel relacionados con el proceso de tercerización logística y, finalmente, d) información detallada de casos de estudio de empresas estadounidenses y europeas. El modelo involucra cinco etapas que se pueden asociar al proceso natural de toma de decisiones para evaluar proveedores (De Boer, 2001). Los pasos y sus características distintivas son los siguientes:

I. Identificar la necesidad de tercerizar funciones logísticas. Los resultados empíricos muestran que la decisión de tercerizar, más que una consecuencia de un análisis de competencias, busca solucionar un problema o aprovechar una oportunidad de negocios. La alta gerencia reconoce, en la mayoría de los casos, esta necesidad o da la autorización a otras áreas funcionales. Aceptada la tercerización, se recomienda integrar un equipo interfuncional y multinivel para continuar con el proceso.

II. Desarrollar alternativas factibles. Esta etapa se identifica como la más difícil sobre todo si la empresa nunca ha hecho tercerización (tarea totalmente nueva caracterizada por la ausencia de información). Los aspectos fundamentales son: estimar los costos de realizar internamente la actividad, precisar los objetivos de la tercerización, expresar estos objetivos en criterios medibles para poder evaluar potenciales candidatos. Para identificar alternativas se recurre tanto a fuentes internas como externas y se sugiere construir árboles de decisión para visualizar diferentes escenarios.

III.Evaluar candidatos y seleccionar proveedor. Se inicia con la definición de criterios, además de los tradicionales de costo, calidad y capacidad de entrega, se considera relevante agregar criterios que tienen que ver con la capacidad del proveedor para desarrollar la relación. Un tamizado inicial basado en juicios y criterios cualitativos (reputación, recomendaciones de clientes) permite elimi- 
nar candidatos no factibles. Los potenciales proveedores son identificados principalmente a través de socios comerciales con experiencia, fuentes menos frecuentes son consultores y agencias especializadas. La evaluación de proveedores está basada en una asignación de pesos relativos a los criterios y la determinación de índices de desempeño por proveedor, para ello se utilizan técnicas cualitativas y se analiza la información que entrega el proveedor en una propuesta formal.

IV.Implantación del servicio. Este paso demanda tres actividades esenciales: a) definir un plan de transición conjunto que especifique los recursos a usar y los compromisos de cada parte, b) proponer un plan de capacitación e intercambio de conocimientos para definir los nuevos procedimientos y prácticas, c) diseñar un plan de adopción en etapas, indicando el orden en que se delegarán actividades, de tal manera que no se ponga en peligro el desempeño.

V. Evaluación continua del servicio. Esta etapa involucra el diseño de medidas conjuntas para evaluar los resultados del servicio, elaboración de políticas de control y mejora, así como una serie de acciones administrativas encaminadas a fortalecer la calidad de la relación.

El modelo propuesto por Bagchi y Virum (BV) está fundamentado en el análisis de diez casos de estudio de empresas involucradas en tercerización estratégica y su enfoque principal es hacia la identificación de las actividades requeridas para que se llegue a la formación de una alianza logística exitosa. El modelo propone tres fases en el proceso de desarrollo de esta alianza logística: a) Reconocer la necesidad de entablar la relación con la tercera parte, b) formación y c) administración de la relación. En la primera fase, el actor principal es la empresa compradora (cliente), la cual debido a factores externos (presión y prácticas administrativas en su ambiente de negocios, o influencia de los proveedores logísticos) o a factores internos (mejorar competitividad, resolver un problema, reducir costos, aumentar eficiencia) reconoce como alternativa a la tercerización. En las siguientes dos fases, tanto empresa como tercera parte son actores activos responsables de la implantación, medición y evolución de la relación. Una serie de actividades y métodos son sugeridos para atender las diversas decisiones implícitas en cada fase; en cuanto a las actividades propuestas tenemos las siguientes: 
1. Definir objetivos para la tercerización y criterios de selección para los potenciales proveedores que estén alineados con estos objetivos. Esta actividad corresponde a la fase II del modelo SL. Al igual que Sink y Langley, se enfatiza la toma de decisiones participativa y la colaboración de diversas áreas funcionales de la empresa en el proyecto.

2. Identificar proveedores calificados, lo que implica un buen conocimiento del mercado de proveedores y el uso de fuentes externas competentes.

3. Articulación de necesidades y deseos, lo que implica la necesidad de obtener información sobre los proveedores no sólo acerca de sus niveles de calidad y capacidad, sino también de su experiencia, reputación, situación financiera, y expectativas de la relación. Éstas son las actividades iniciales que el modelo SL propone en la fase III.

4. Evaluación y selección de proveedores con la sugerencia específica de aplicar el proceso de Jerarquización Analítica (AHP en inglés) como método viable para jerarquizar las propuestas de los proveedores candidatos. La fase de tamizado que propone SL no está considerada, lo que sugiere que se ha aplicado el principio de satisfacción; esto es, que el número de propuestas recopiladas es un conjunto que satisface las necesidades del grupo decisor.

5. Desarrollo de un plan de integración, el cual debe contemplar el intercambio e integración de conocimientos y tecnología, la estrategia de coordinación y la asignación de responsabilidades. Corresponde a la fase IV del modelo SL.

6. Creación de una relación ganar-ganar a través de compartir expectativas, hacer retroalimentación y dar apoyo para mejoras conjuntas. Esta importante actividad que contribuye al desarrollo de la confianza y el compromiso entre las partes involucradas no está considerada en el modelo SL.

7. Evaluar y analizar el desempeño, además de reconocer la importancia de medidas compartidas por el cliente y la tercera parte (establecido en el modelo $\mathrm{SL}$ ), se insiste en el diseño de medidas correctas y suficientes.

8. Redefinir metas y objetivos que reflejen no sólo la experiencia obtenida de la relación, sino las demandas del mercado y el compromiso para un proceso de mejora continua de la relación. Se identifica como factor crítico el que la ter- 
cera parte sea capaz de desarrollar capacidades adicionales que le permitan ampliar el servicio que ofrece y con ello aumentar el valor de la relación establecida.

Si bien los modelos anteriores derivan de la información obtenida sobre las decisiones de tercerización empresariales, esta información es específica al contexto de empresas en países desarrollados en los cuales está más difundida la práctica de tercerización y se tiene un mercado de proveedores logísticos competitivo y bien estructurado (Arroyo et al., 2004).

El presente trabajo explora el proceso de toma de decisiones en tercerización para empresas maduras establecidas en México con el propósito de aumentar el conocimiento sobre el proceso de selección de proveedores y el impacto que el contexto de negocios y las políticas empresariales tienen sobre los factores que se toman en cuenta al constituir relaciones con terceras partes. Para atender a este propósito se analizan relaciones con diferentes periodos de duración (desde un semestre hasta quince años) y que han sido calificadas como exitosas por la empresa compradora. El proceso de formación de cada relación se contrasta con las recomendaciones de los modelos normativos/prescriptivos discutidos previamente para identificar acciones administrativas que sean específicas al contexto mexicano y que aun cuando se desvíen de lo que se consideran las mejores prácticas han permitido el desarrollo de relaciones exitosas. La naturaleza de la investigación es exploratoria porque se orienta a determinar qué recursos utilizan las empresas en México para tomar decisiones relacionadas con el proceso de subcontratación de actividades logísticas para así — a través de la información detallada que se recopile - identificar prácticas habituales que se califiquen como exitosas y sugerir herramientas formales de decisión que puedan ser aplicadas para mejorar las actividades del proceso de tercerización.

Los modelos de Sink y Langley (SL) y el de Bagchi y Virum (BV) constituyen la referencia básica para analizar las relaciones estudiadas. Ambos modelos asumen que la alternativa de tercerización es aceptable y que hay un mercado competitivo de operadores logísticos para elegir, lo que contrasta con los modelos de Lonsdale y McIvor, los cuales hacen mayor énfasis en la evaluación de la decisión de tercerizar enfatizando el riesgo implícito en esta decisión, por lo que proponen considerar no sólo la competencia del mercado de terceras partes, sino también su estructura (monopolio-oligopolio vs. mercado competitivo) y reconocen como al- 
El proceso de toma de decisiones para la tercerización de funciones logísticas:

prácticas mexicanas versus mejores prácticas establecidas

ternativa el desarrollo de proveedores internos. Esta etapa inicial - evaluación de la decisión de tercerizar determinada actividad logística - se identifica como un paso crítico en el ambiente mexicano y, por tanto, es considerada en el análisis de los casos presentados. En la tabla 1 se resumen y contrastan los cuatro modelos, indicándose las acciones y métodos sugeridos para apoyar cada etapa. Algunas de las etapas (búsqueda y selección de proveedores) involucran varias subetapas, por lo cual se presenta una división adicional dentro del cuadro comparativo.

Tabla 1

\section{Resumen de los modelos de decisión para el proceso de tercerización}

\begin{tabular}{|c|c|c|c|c|}
\hline \multirow{2}{*}{$\begin{array}{l}\text { Etapas del } \\
\text { procese de } \\
\text { tercerizacibn }\end{array}$} & \multicolumn{4}{|c|}{ MODELOS } \\
\hline & Bagchi y Virun & Sink y Langley & Lotsdale & Melver \\
\hline $\begin{array}{l}\text { E.tapa } 1 \\
\text { Reconocimiesto } \\
\text { de la necesidad } \\
\text { de lereiarizar }\end{array}$ & $\begin{array}{l}\text { Metivadar de la } \\
\text { Aercerisación }\end{array}$ & 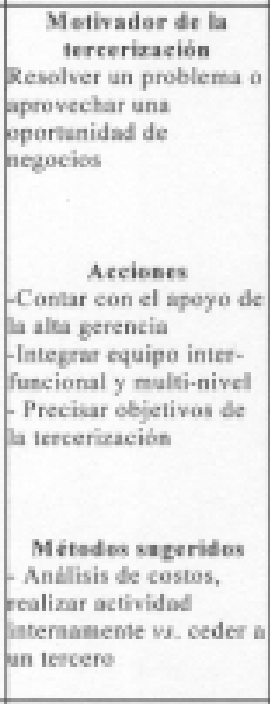 & $\begin{array}{l}\text { Mativador de la } \\
\text { tercerizacibn } \\
\text { Posibilidad de } \\
\text { mejorar la } \\
\text { posición } \\
\text { pompelitiva } \\
\\
\text { A eciones } \\
\text { Clasificar la } \\
\text { actividad por la } \\
\text { imporiancia que } \\
\text { cepecoenta para la } \\
\text { compania. }\end{array}$ & $\begin{array}{l}\text { Metivader de la } \\
\text { eerecrizaciba } \\
\text { Mayor eficiencia } \\
\text { pi la actividad es } \\
\text { realicada por } \\
\text { pgentes exicmos }\end{array}$ \\
\hline $\begin{array}{l}\text { Etapa } 2 \\
\text { Bdscqueday } \\
\text { weleceión de } \\
\text { proveedores } \\
\text { 2.1. Bùsqueda de } \\
\text { provecdores }\end{array}$ & 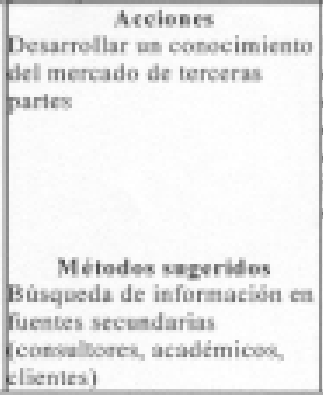 & $\begin{array}{l}\text { Aceieses } \\
\text { Identificar provecdores } \\
\text { con base en su reputa- } \\
\text { ción en el nector de la } \\
\text { empeca y el mercado } \\
\text { de 3PL (referencias } \\
\text { interass y esternas a la } \\
\text { erganización) }\end{array}$ & $\begin{array}{l}\text { Aecioaes } \\
\text { Analixis del } \\
\text { mereado de } \\
\text { proverdores y } \\
\text { esiablecimiento } \\
\text { de se competencia }\end{array}$ & $\begin{array}{l}\text { Aecieses } \\
\text { Analisis de } \\
\text { fapacidades } \\
\text { fenicas y de } \\
\text { pelación de } \\
\text { proveedores }\end{array}$ \\
\hline
\end{tabular}


Tabla 1 (continuación)

\section{Resumen de los modelos de decisión para el proceso de tercerización}

\begin{tabular}{|c|c|c|c|c|}
\hline $\begin{array}{l}\text { 2.2. Preseleccibe } \\
\text { provededes }\end{array}$ & \begin{tabular}{|c|} 
Acciones \\
Oteener inscemacion \\
pobre proveedores
\end{tabular} & $\begin{array}{l}\text { Aeciones } \\
\text { Evaluación cualitativa de entre } \\
6-8 \text { proveedores } \\
\text { Métodos sugeribles } \\
\text { - Preguntas abiertas a } \\
\text { provoodores y definición de } \\
\text { piveles de requacich } \\
\text { - Construoción de ártoles de } \\
\text { decisión }\end{array}$ & \begin{tabular}{|l|}
\multicolumn{1}{|c|}{ Acciones } \\
Evalur riesgo \\
si el mercado \\
es moenpolio uf \\
oligopolio
\end{tabular} & \begin{tabular}{l}
\multicolumn{1}{c}{ Actiones } \\
Andlisis de las \\
capocidades técnicare \\
desputs de las \\
cagecidades de \\
relación
\end{tabular} \\
\hline $\begin{array}{l}\text { 2.3. Selección de } \\
\text { proveedores }\end{array}$ & \begin{tabular}{|l|}
\multicolumn{1}{|c|}{ Aceiones } \\
- Definición de \\
criterios de selección \\
- Visitas in situ y \\
entrevistas a \\
proweedores
\end{tabular} & 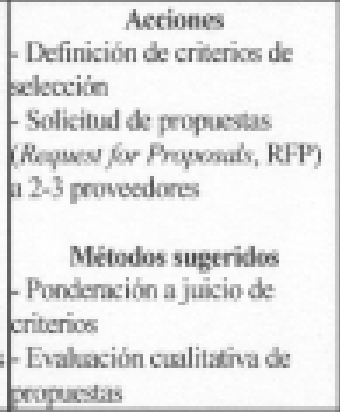 & - & \begin{tabular}{l}
\multicolumn{1}{c}{ Actiones } \\
Definición de \\
criterios đécnicos, \\
ocmerciales y de \\
pelación para la \\
eclección
\end{tabular} \\
\hline \begin{tabular}{|l|} 
Etapa 3 \\
indrumentación \\
de la relación
\end{tabular} & 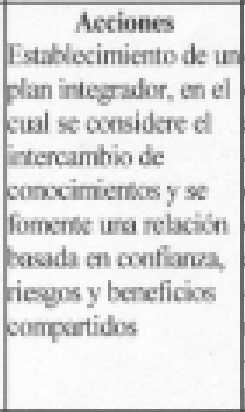 & 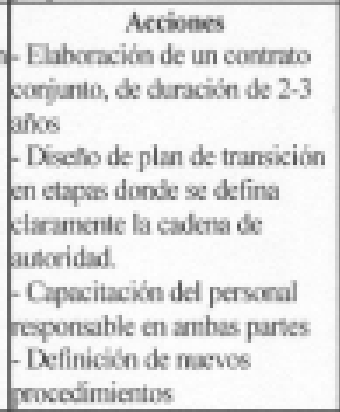 & - & - \\
\hline $\begin{array}{l}\text { Etapa } 4 \\
\text { Evaluacikn y } \\
\text { control }\end{array}$ & \begin{tabular}{|l|} 
Acciones \\
- Evaluar el \\
desempeho sobre la \\
base de scrvicio al \\
cliente \\
- Redefinir metas y \\
objetivos después de \\
la evalusación
\end{tabular} & 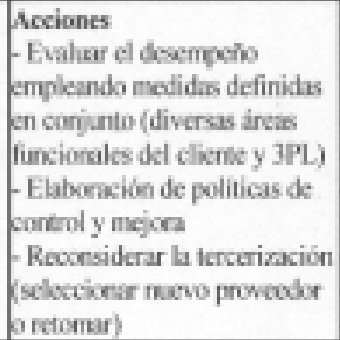 & $\begin{array}{l}\text { Acciones } \\
\text { Definir un } \\
\text { responsable } \\
\text { que administre } \\
\text { el descmpeño } \\
\text { enfocindose a } \\
\text { reducir el } \\
\text { oportunismo } \\
\text { exosiracousal y la } \\
\text { complasencia }\end{array}$ & 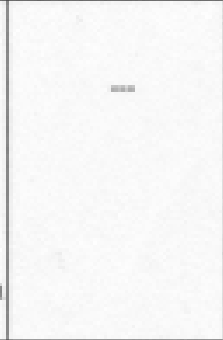 \\
\hline
\end{tabular}


El proceso de toma de decisiones para la tercerización de funciones logísticas:

prácticas mexicanas versus mejores prácticas establecidas

\section{Metodología y descripción de unidades de estudio}

Dado que se requiere ahondar en los detalles del proceso real de toma de decisiones sobre tercerización y documentar con detalle las varias etapas del proceso, el método elegido para realizar la indagación fue el de caso de estudio (Yin, 2003). Debido a las dificultades de lograr la participación de las empresas para aportar los pormenores de sus procesos internos asociados a la tercerización, la investigación se concentró en cuatro empresas con las cuales se tenía establecido algún contacto personal. Estas cuatro empresas fueron elegidas porque recientemente (menos de dos años) estuvieron involucradas en la revisión o arranque de un proyecto de tercerización de actividades logísticas diversas y todas juzgan exitosa la relación establecida. La información deseada se obtuvo a través de una serie de entrevistas semi-estructuradas con los participantes en el proceso de toma de decisiones, incluyéndose a algún administrativo de alto nivel (gerente comercial o de logística) cuya recomendación tuvo un peso importante sobre la decisión final. Por motivos de confidencialidad, los datos secundarios en forma de documentos o reportes estuvieron limitados a la información que los participantes entregaron como datos adicionales, por lo cual esta información se considera más bien complementaria que relevante para realizar una validación de los datos obtenidos en las entrevistas. La guía para las entrevistas fue derivada de las etapas identificadas para el proceso de tercerización; durante la entrevista se solicitó a los tomadores de decisiones que explicaran con detalle las acciones y métodos que utilizaron para completar las etapas fundamentales del proceso.

Las entrevistas fueron grabadas y transcritas, este material fue analizado por separado por los participantes del proyecto para después ser revisadas por el grupo como forma de validez cruzada para la interpretación de la información. Los cuatro casos involucraron a empresas compradoras de tamaño grande (de más de 500 empleados) con subsidiarias en otros países y localizadas en los parques industriales de la ciudad de Toluca.

La primera empresa (E1) es una compañía multinacional con corporativo en Alemania, fabricante de una amplia línea de productos automotrices y con varios años de operación en México. Esta empresa ha tercerizado desde 1996 las actividades de manufactura y ensamble de productos además de servicios complementarios (limpieza, jardinería, seguridad, etc.), aunque la tercerización de funciones logísticas es reciente. El proceso de tercerización estudiado fue para una actividad de logística reversa: la recuperación de envases retornables; esta actividad era realizada originalmente por un proveedor interno, con el cual se tuvieron múltiples 
problemas de calidad en el producto (empaque retornado sucio o dañado), mala administración de su inventario y deficiencias en el nivel requerido de stock de seguridad. La empresa intentó como primera solución desarrollar otros proveedores internos (nacionales) sin éxito y con un mayor costo, por lo cual la alta gerencia consideró como alternativa la tercerización, esperando que un proveedor externo (3PL) pudiera lograr la eficiencia requerida para esta actividad de logística reversa. Un equipo multifuncional fue integrado (áreas de compra y logística) para realizar la búsqueda y selección del proveedor. Durante la etapa de búsqueda de proveedores, la alta gerencia solicitó que además de la recuperación del empaque retornable se identificaran proveedores que en un plazo mediato pudieran hacerse cargo también de sacar el producto terminado de las líneas de producción, almacenarlo y distribuirlo, pues se pronosticó que la capacidad de almacén de la empresa quedaría excedida en 2-3 años y la alta gerencia había decidido no invertir más en capacidad de almacén ni gastos de distribución, sino destinar el espacio disponible para ampliar la línea de productos que fabrica esta planta. La política de adquisiciones de la empresa exige elegir entre tres opciones, por lo cual el grupo responsable de la decisión seleccionó y precalificó a varios candidatos antes de solicitar cotizaciones a los mejor evaluados y tener disponibles tres propuestas completas. El departamento de logística estaba en la etapa final de seleccionar entre estos tres candidatos al proveedor para el servicio de recuperación de envase retornable y del manejo de producto terminado, cuando solicitó asesoría técnica a los dos primeros autores de este estudio, quienes propusieron el uso de métodos de superación para realizar la selección del proveedor. La empresa acordó un contrato con el proveedor seleccionado a través de esta metodología; al momento de la realización de este estudio, casi se había completado la fase de implantación de la relación y se estaba procediendo con la evaluación de resultados.

El segundo caso involucra a una empresa (E2) que es proveedor interno de la compañía líder en panificación en México, la cual es reconocida por sus prácticas de distribución en el mercado de bienes de consumo. Esta empresa - que fabrica los exhibidores y anaqueles para todos los productos de la empresa - contrató los servicios de una tercera parte para administrar el proceso de búsqueda y selección de proveedores de materias primas a través de subastas en línea, lo cual efectuó atendiendo la solicitud de su corporativo. El proveedor logístico es una empresa con capital latinoamericano que fue elegida por un grupo de empresas líderes en productos de consumo debido a su experiencia internacional en el empleo de sistemas en línea para búsqueda y selección de proveedores de productos y materias primas. Durante la búsqueda y selección de esta tercera parte, el grupo de ejecutivos 
El proceso de toma de decisiones para la tercerización de funciones logísticas:

prácticas mexicanas versus mejores prácticas establecidas

de alto nivel de las empresas de bienes de consumo sólo consideraron operadores internacionales porque en el mercado local no se tenían referencias de operadores de prestigio. Varias de las empresas integrantes de esta gran compañía de panificación tenían ya contratos con el operador logístico cuando la alta gerencia recomendó a la empresa fabricante de exhibidores que analizara la opción de tercerizar a esta compañía la actividad de búsqueda y selección en línea de sus proveedores de materia prima. El gerente de logística fue el responsable de tomar la decisión; además de la recomendación directa del corporativo de la empresa, el otro elemento crítico considerado en la decisión, fue la propuesta de la 3PL de que el pago de sus servicios sería un porcentaje de los ahorros registrados por la empresa. Además de los servicios de administración de la subasta en línea, la 3PL apoya a la empresa con consultoría para la negociación y delineación de contratos con los proveedores seleccionados. El proyecto de tercerización tiene ya varios años de implementado y ha derivado en ahorros sustanciales para la empresa.

La empresa del caso tres (E3) es otra multinacional que pertenece a un grupo industrial que manufactura productos de marca tanto para el mercado industrial como para el de consumo. El grupo está integrado por cuatro sectores de negocios: detergentes y cuidado del hogar, cosméticos y productos de limpieza, adhesivos y desarrollos técnicos. El corporativo de esta multinacional también está ubicado en Alemania y la empresa está en proceso de incrementar su presencia en otros países (actualmente, hay subsidiarias en 125 países). Esta compañía registra un buen crecimiento en sus cuatro sectores, especialmente en el de adhesivos. En México, el grupo está consolidando su posición a través de la adquisición de marcas bien establecidas y cuenta con plantas localizadas en los estados de México y Guanajuato. En su misión, el corporativo indica que la capacidad medular (core) del grupo es el desarrollo de marcas. Después de un análisis estratégico de la posición de la empresa en México, la alta gerencia decidió concentrar sus recursos en el desarrollo de esta capacidad y emplear los servicios de terceros para la realización de actividades no-medulares. Los directores de los departamentos de operaciones, marketing y comercial realizaron un análisis económico de sus procesos administrativos y decidieron que la distribución de productos era uno de los procesos logísticos que debía tercerizarse. Un conjunto de operadores logísticos registrados en directorios especializados fue seleccionado para solicitarles cotizaciones formales. Los proveedores fueron evaluados bajo múltiples criterios, de los mejor calificados se obtuvo además información directa de los clientes a los que atienden. El operador seleccionado a través de este proceso 
realiza servicios de cross-docking, la entrega de productos a los distribuidores mayoristas de la empresa, y supervisa que éstos realicen la distribución y promoción de los productos a los comercializadores al menudeo. El proyecto de tercerización ya ha sido implementado y al momento del estudio se realizaba la primera evaluación de los resultados.

La empresa del caso cuatro (E4) es de origen estadounidense y fabrica productos en dos líneas básicas: cosméticos y plásticos; los productos son promocionados y distribuidos en forma directa por agentes independientes. Las órdenes semanales se envían a 130 distribuidores regionales que los entregan a estos agentes de ventas. La empresa renueva periódicamente sus productos y distribuye los catálogos correspondientes a los agentes de ventas. La planeación de la producción depende de la disponibilidad para usar los moldes que constituyen el activo tecnológico principal de la empresa, manteniéndose por tanto un alto inventario. La distribución ha estado siempre a cargo de una tercera parte desde que la empresa inició actividades en México (quince años desde la decisión) debido a que esta actividad logística nunca ha figurado entre las competencias - desarrollo de moldes y manufactura son las principales - de la empresa. Para hacerse cargo de la distribución se tiene establecido un contrato tácito - basado en acuerdos verbales entre las partes - con un proveedor en particular que es el líder en el mercado de transportistas. Este proveedor tiene asignado la mayoría del volumen y se mantiene con él una relación de confianza mutua y para la que no se contempla una fecha de terminación. Sin embargo, anualmente se hace una revisión del contrato en la que intervienen el director general de planta, el gerente de logística y el director de tráfico con el fin de re-negociar los costos del servicio con este proveedor principal, quien en términos de desempeño está altamente calificado. De acuerdo con la evaluación, se revisa la base actual de transportistas minoritarios o bien se reasigna el volumen de distribución. Operar con varios transportistas es la forma que la empresa eligió para reducir el riesgo de la tercerización a un único proveedor y contar con un margen de negociación en relación con los costos del servicio.

\section{Resultados y discusión}

En la tabla 2 se describen los elementos más relevantes de los procesos de tercerización analizados. Para la primera etapa del proceso (Reconocimiento de la necesidad de tercerizar), además de darse una definición concisa del problema administrativo por atender, se tipifica el tipo de problema de acuerdo con la cla- 
sificación de situaciones típicas de compra (purchasing) sugeridas por De Boer et al. (2001). Los casos 2 y 4 se identifican como recompra modificada, puesto que para el caso 2, si bien la empresa pretende iniciar el proyecto de tercerización, la 3PL en cuestión ya presta servicios logísticos a empresas hermanas de E2 y conoce los requerimientos del corporativo y la forma de operación del grupo. Además la empresa del caso 2 cuenta con información de primera mano que las compañías de su mismo grupo industrial tienen respecto al desempeño de la tercera parte, lo que permite una evaluación más objetiva y rápida del atractivo de la tercerización y del potencial para establecer con este proveedor una relación de asociación. Para la empresa del caso 4, la recompra modificada ocurre debido a que no sólo hay que reasignar y renegociar con proveedores existentes la distribución de los productos que fabrica $\mathrm{E} 4$, sino que puede requerirse identificar, evaluar y seleccionar nuevos proveedores; para los otros dos casos el problema involucra un nuevo proyecto para la empresa, pero las situaciones de decisión difieren respecto a la complejidad de la actividad logística que va a ser cedida. En relación con el caso 3 la actividad está bien definida y si bien involucra varias actividades, todas son rutinarias (distribución); en tanto en el caso 1, la actividad tercerizada es compleja porque demanda acciones y procedimientos novedosos para resolver los problemas de servicio.

Con base en los hallazgos de los casos de estudio, se derivan una serie de proposiciones que permiten: a) establecer si las acciones y métodos sugeridos por los modelos normativos/prescriptivos presentados previamente fueron empleados por los tomadores de decisiones en los cuatro procesos de tercerización analizados o b) identificar las prácticas observadas y analizar su conveniencia. En caso de detectarse errores en los procedimientos aplicados, se revisó la literatura sobre métodos de toma de decisiones para elaborar propuestas de los métodos que podrían emplearse para mejorar etapas específicas del proceso de tercerización. 
Tabla 2

\section{Hallazgos de las entrevistas para los casos de estudio}

\begin{tabular}{|c|c|c|c|c|}
\hline $\begin{array}{l}\text { Elapas del procke de } \\
\text { inwerirarile }\end{array}$ & Cave 1 & Caie? & Can 3 & Cones 4 \\
\hline 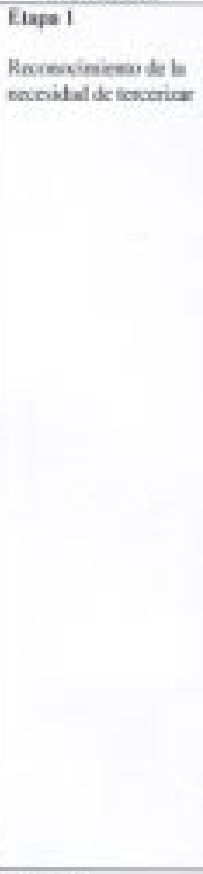 & 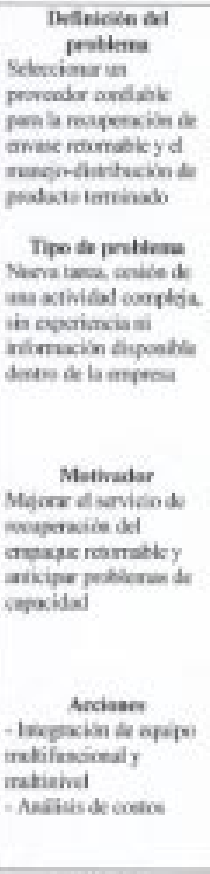 & 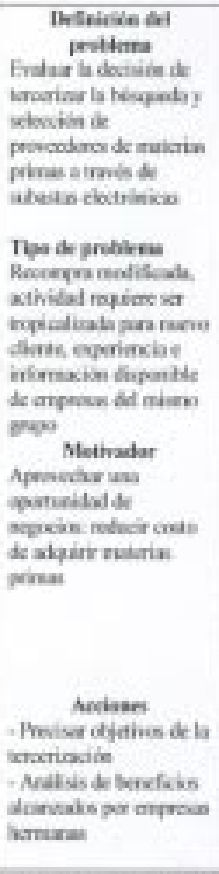 & 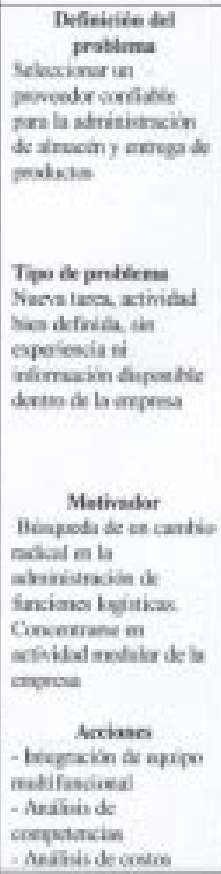 & 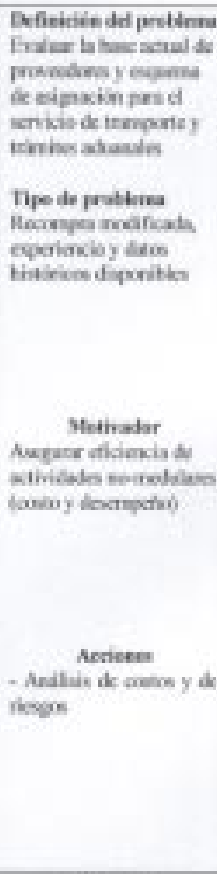 \\
\hline 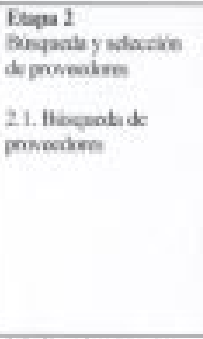 & 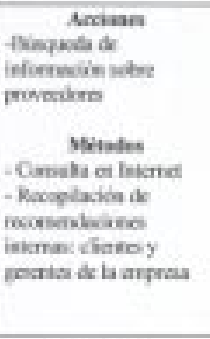 & 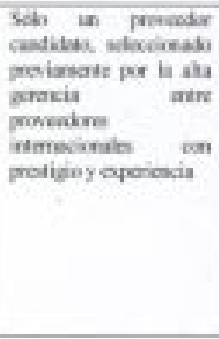 & 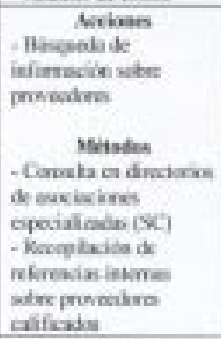 & 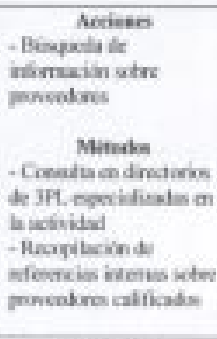 \\
\hline 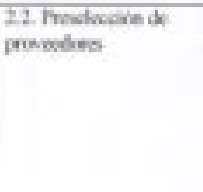 & 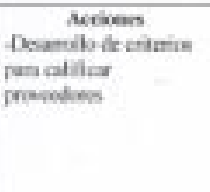 & & 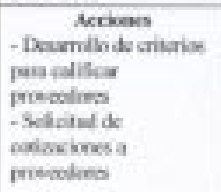 & 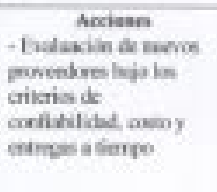 \\
\hline
\end{tabular}

continúa... 
El proceso de toma de decisiones para la tercerización de funciones logísticas:

prácticas mexicanas versus mejores prácticas establecidas

Tabla 2 (continuación)

Hallazgos de las entrevistas para los casos de estudio

\begin{tabular}{|c|c|c|c|c|}
\hline & 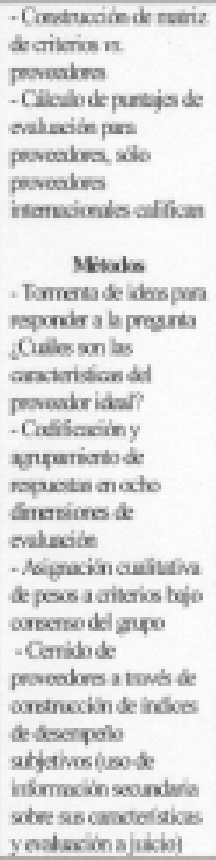 & & 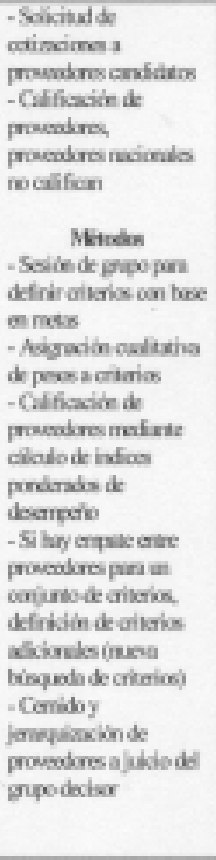 & 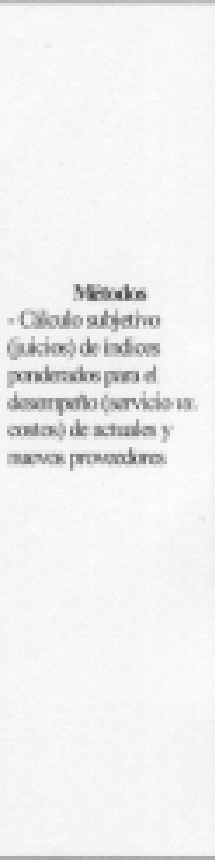 \\
\hline $\begin{array}{l}\text { 2.3. Sckocinid } \alpha \\
\text { peveades }\end{array}$ & 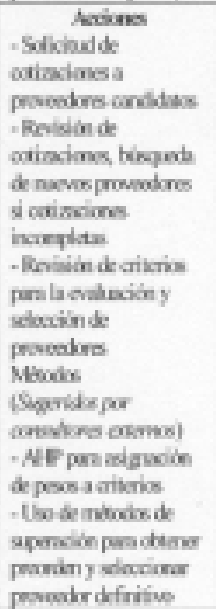 & 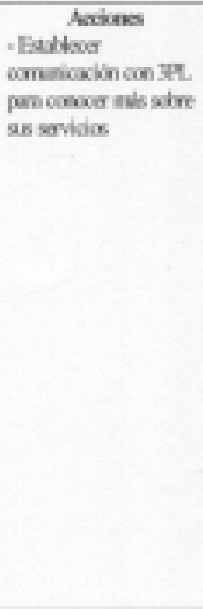 & 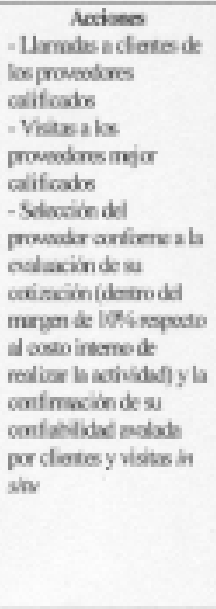 & 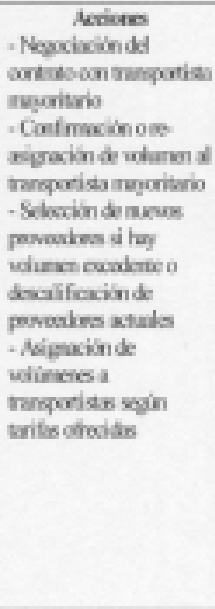 \\
\hline
\end{tabular}

continúa... 


\section{Tabla 2 (continuación) \\ Hallazgos de las entrevistas para los casos de estudio}

\begin{tabular}{|c|c|c|c|c|}
\hline Etapa 4 & 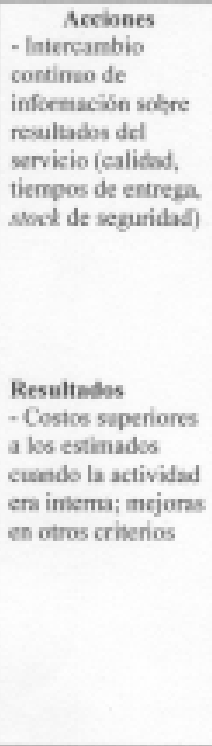 & 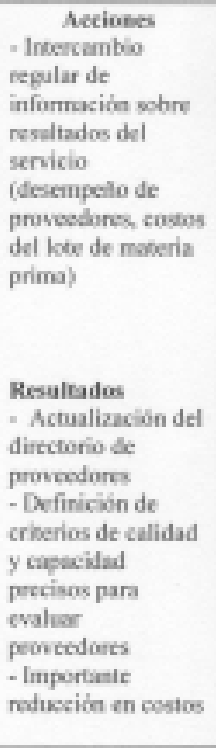 & $\begin{array}{l}\text { Resultades } \\
\text { - Nivel de sernicio } \\
\text { del } 98 \% \text { de acuendo } \\
\text { con indicadores } \\
\text { internos de la } \\
\text { empresa } \\
\text { - Coelos de ta } \\
\text { actividad dentro de } \\
\text { los mulrgenes } \\
\text { establecidos al } \\
\text { tercerizar }\end{array}$ & 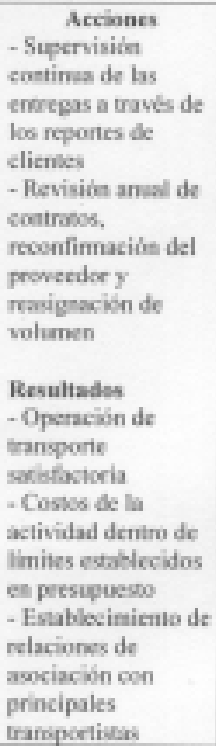 \\
\hline
\end{tabular}

\section{Proposiciones}

- La decisión de tercerizar actividades logísticas obedece a motivos diversos que incluyen la resolución de un problema (caso 1), el aprovechar una oportunidad de negocios (caso 2) o mejorar la posición competitiva de la empresa al ceder a terceros actividades no consideradas medulares (casos 3 y 4). La decisión de tercerizar es identificada por la alta gerencia, cuya participación es sólo marginal en etapas posteriores del proceso.

- La viabilidad de la tercerización se analiza principalmente sobre la base de los costos de realizar la actividad internamente, en ningún caso el costeo fue de tipo ABC como se sugiere en la literatura. Las empresas están dispuestas a aceptar un cierto incremento en los costos al subcontratar la actividad a cambio de mejoras en el servicio a sus clientes y la liberación de recursos (físicos y humanos) que se puedan destinar para el desarrollo de las actividades medulares de la empresa. 
- No se practica un benchmarking con las empresas del sector para identificar aquellas actividades logísticas básicas que están dejando de ser realizadas por la empresa y asignadas a terceros ni se realiza un análisis de eficiencia para evaluar el desempeño interno.

- Para las subsecuentes etapas del proceso de tercerización se integran equipos multifuncionales y multinivel (caso 1 y 3 ) coordinados por gerentes de operaciones, de logística o generales.

- El mercado nacional de 3PL no se considera competente para desarrollar actividades complejas; la única actividad asignada a un proveedor nacional es simple porque implica el transporte más el trámite aduanal y la generación de documentos básicos. Las empresas administran su riesgo de tercerizar al buscar proveedores con prestigio y experiencia internacional, conseguir referencias internas, realizar visitas a proveedores potenciales y al verificar la información recopilada en fuentes secundarias o en las cotizaciones que remiten los proveedores realizando visitas a los clientes de la 3PL.

- El número de proveedores por incluir en la etapa de calificación se rige por el principio de satisfacción, el cual se entiende como sigue: se incluyen tantos proveedores como el grupo decisor considere conveniente, cuando el grupo inicial no resulta bien calificado, se amplia la búsqueda hasta que el grupo decisor está satisfecho con el número de proveedores que ha evaluado. Los criterios en la etapa de calificación son generales y se utiliza información secundaria (reportes, directorios, páginas web) para calificar a los candidatos. Estas acciones empatan cercanamente las sugeridas por Sink y Langley (1997).

- El total de proveedores que se considera en la etapa de selección queda a criterio del grupo o bien se define por políticas de la empresa. En esta etapa se definen criterios más precisos entre los cuales el costo está entre los más importantes. La empresa del caso 1 incluyó todos los criterios pertinentes para hacer la evaluación de los candidatos, en tanto la del caso 3 inició con pocos criterios y procedió a incrementar su número para desempatar evaluaciones. Estos dos esquemas de trabajo han sido reportados en la literatura sin una conclusión sobre cual de los dos es una mejor práctica (De Boer, 2004). Las cotizaciones que remiten los proveedores son requeridas para completar esta 
etapa de selección, pero la solicitud de cotizaciones en el formato de RFI (Request For Information) puede realizarse desde la etapa de búsqueda y calificación de proveedores.

- Un análisis detallado de las capacidades de relación con proveedores candidatos no fue evidente en los casos, sin embargo para los casos 1 y 2, el grupo o individuo decisor manifestó preferencia por tratar con 3PL que tuvieran como clientes empresas de su mismo sector. De acuerdo con las declaraciones de los entrevistados estas referencias les garantizan que las 3PL's conocen la "cultura" del sector y eso facilita el trabajar con ellos.

- En la etapa de selección de proveedores se consideran múltiples criterios que cubren las dimensiones de confiabilidad del servicio, costo, capacidad y experiencia. La práctica es llegar a un consenso por parte del grupo decisor con respecto a los pesos (grados de importancia) que se asignarán a cada criterio; establecidos los pesos se calculan promedios ponderados para determinar el nivel de desempeño de los proveedores.

- Aun cuando las empresas participantes son líderes dentro de su sector, no utilizan ni conocen métodos formales para la evaluación de alternativas y la construcción de una jerarquía de proveedores. La selección se basa en costos dado un nivel aceptable de otros criterios (caso 4) o en la suma ponderada de los atributos para los proveedores (casos 2 y 3). Los métodos de superación empleados para seleccionar al proveedor de la empresa en el caso 1 fueron sugeridos por los autores de este trabajo y resultaron bien aceptados y apreciados por el grupo decisor. Por otra parte, las empresas de los casos 3 y 4 consideraron satisfactorio su procedimiento de evaluación/selección actual porque han obtenido resultados satisfactorios de la tercerización. Cabe aclarar que para estas empresas, los servicios logísticos tercerizados (distribución y transporte) se clasifican como básicos, esto es relativamente estandarizados, con requisitos operativos bien definidos y estables (Andersson y Norrman, 2002) lo que facilita la comparación de alternativas.

- Los contratos legales son el mecanismo básico para el control de la relación y en ellos se incluyen condiciones específicas para los resultados de la actividad subcontratada (la propuesta inicial la hace el cliente y se negocia con la 3PL) y cláusulas de penalización. Los contratos se revisan periódicamente (plazos 
El proceso de toma de decisiones para la tercerización de funciones logísticas:

prácticas mexicanas versus mejores prácticas establecidas

menores de un año) ante la imposibilidad de establecer a priori todas las condiciones para la relación, a menos que se trate de actividades básicas para las cuales se cuente con información suficiente como para establecer desde un inicio los compromisos de empresa y 3PL (caso 4). Los acuerdos tácitos ocurren sólo después de un periodo largo de operación, pero aún en estos casos, el riesgo se apalanca estableciendo relaciones con otros proveedores (caso 4).

- El plan para la transferencia de la actividad logística considera el intercambio de conocimientos entre las dos partes y la definición del personal de enlace o responsable de supervisar el desempeño de la actividad. La pronta resolución de conflictos, la flexibilidad en la evaluación y la tolerancia de ambas partes mientras ocurre la transición son factores críticos para completar esta etapa. De acuerdo con Moore (1998), la ausencia de conflictos influye en la efectividad y consolidación de la relación más que los eventos positivos que se deriven. En particular para el caso 1, se pudo constatar la importancia que tiene sobre la percepción de la efectividad de la relación la pronta solución de conflictos y la intervención de mandos superiores en caso de problemas.

- Tanto para relaciones de tercerización recién creadas como para aquellas que ya tienen años de establecidas, se da un intercambio continuo de información tanto por mecanismos formales como informales con intervención de los mandos superiores sólo en caso de conflictos o situaciones imprevistas. La obtención de beneficios en el corto plazo (reducción de costos para la empresa del caso 2 y mejoras en el servicio para los otros casos) y la ausencia de acciones oportunistas por parte de los proveedores (en el caso 1 la tercera parte incluso realizó unilateralmente la inversión requerida para atender al comprador) deriva en una percepción de efectividad de la relación y un interés por continuarla de forma indefinida (casos 2 y 4 están en esta situación de avance). La relevancia de los citados factores en la evaluación de resultados para la tercerización está de acuerdo con los estudios empíricos reportados en la literatura (Moore, 1998 y Knemeyer y Murphy, 2004).

- Aun cuando se hayan empleado métodos a juicio y búsquedas parciales durante el proceso de tercerización, las cuatro relaciones analizadas se evalúan como exitosas, aún las de reciente creación, lo que se asocia a las motivaciones de los participantes para lograr eficiencia en las actividades logísticas. La evidencia de los casos confirma la conclusión establecida en el estudio de Kannan y 
Choon Tan (2002) relacionado con la influencia determinante que tiene el compromiso estratégico de los proveedores en el desempeño: las 3PL de los casos 1, 2 y 3 consolidan su posición en el mercado mexicano a través de las relaciones de tercerización (para el caso 1 resulta ser su oportunidad de entrada) y para la 3PL del caso 4, el cliente contribuye a su credibilidad y prestigio.

Con base en los cuatro modelos considerados en este trabajo y los resultados del análisis de los procesos de tercerización en el contexto mexicano, se sugieren acciones y métodos para apoyar cada una de las etapas del proceso. Por conveniencia, esta propuesta se condensa en la tabla 3 en la cual se hace una distinción entre las actividades nueva tarea / no información disponible y aquellas que involucran re-compra modificada e información disponible. Para varios de los métodos cuantitativos sugeridos, se cuenta con referencias en la literatura que avalan su aplicabilidad (las referencias correspondientes se incluyen en la misma tabla). 
El proceso de toma de decisiones para la tercerización de funciones logísticas:

prácticas mexicanas versus mejores prácticas establecidas

Tabla 3

Acciones y métodos para facilitar el proceso de tercerización

\begin{tabular}{|c|c|c|}
\hline & Nava tasa / Doss rodeprithos & 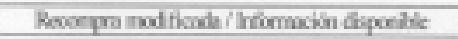 \\
\hline 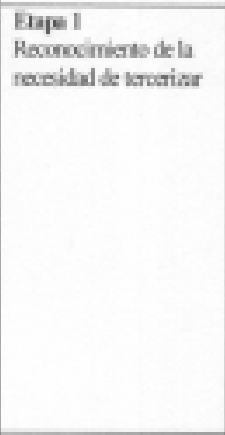 & 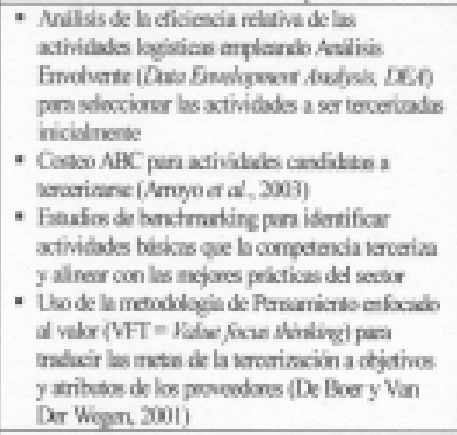 & 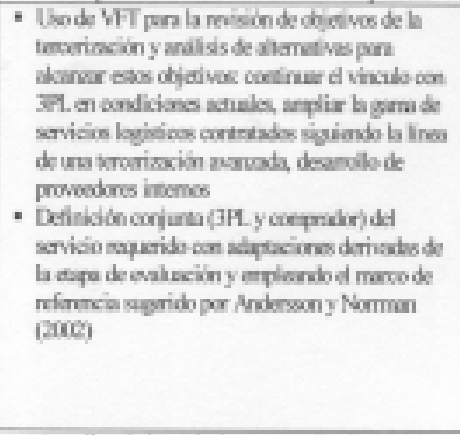 \\
\hline $\begin{array}{l}\text { Etape } 21 \\
\text { Biccuata } \\
\text { povedores }\end{array}$ & 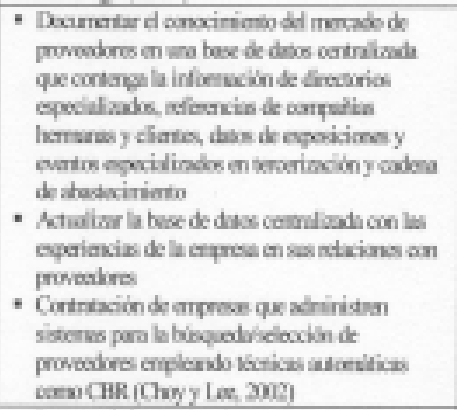 & 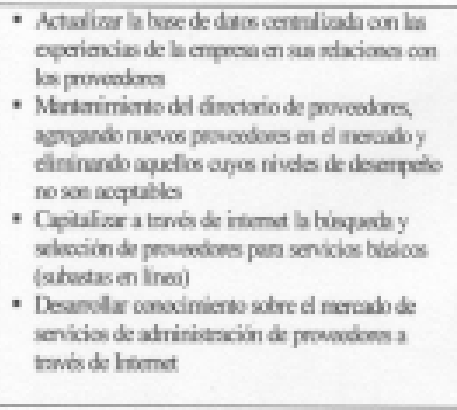 \\
\hline Deapa 2.2 Prescloockin & 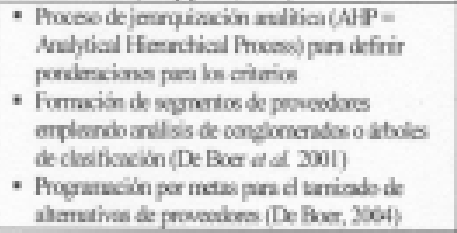 & 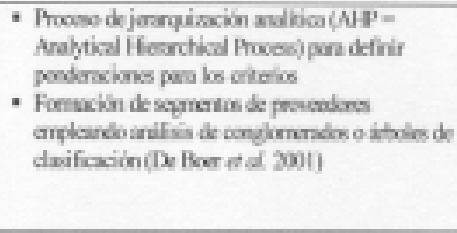 \\
\hline $\begin{array}{l}\text { Drapin } 25 \\
\text { Scladion } \\
\text { provocikes }\end{array}$ & 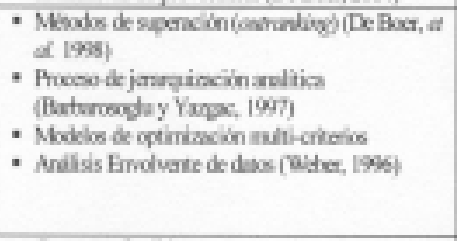 & 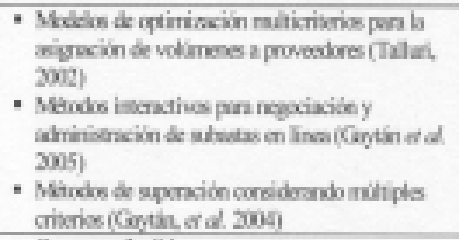 \\
\hline $\begin{array}{l}\text { Elape } 3 \\
\text { ledumentaion de in } \\
\text { rdacide }\end{array}$ & 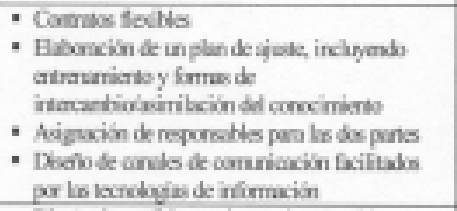 & 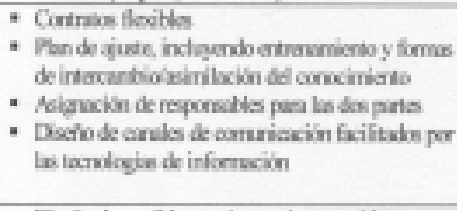 \\
\hline $\begin{array}{l}\text { Etapa } 4 \\
\text { Eveluxion y oonod }\end{array}$ & 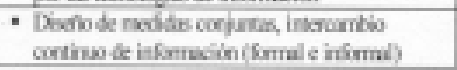 & 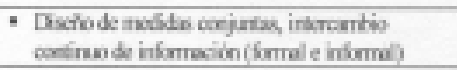 \\
\hline
\end{tabular}




\section{Conclusiones}

En los cuatro casos estudiados se realizó un proceso de decisión objetivo para definir la actividad logística más conveniente de tercerizar. La selección estuvo motivada por la ocurrencia de problemas o detección de oportunidades de negocios y no por un análisis enfocado a determinar la contribución de la tercerización logística para obtener una cadena de suministros más eficiente o flexible. Se recomienda abrir el conjunto de alternativas a otras funciones para las cuales puede haber un mercado de proveedores más competitivo.

La decisión de qué actividad logística ceder a terceras partes se toma en el nivel corporativo transfiriéndose la selección de la tercera parte a los niveles operativos. La práctica de integrar un equipo multi-funcional y multi-nivel responsable del proceso de tercerización contribuye a establecer objetivos para la tercerización que satisfagan a todas las áreas de la empresa.

No hay una recomendación formal respecto a cuál procedimiento seguir para evaluar proveedores potenciales: a) incluir un gran número de características o b) evaluar empleando pocos criterios clave. En términos del esfuerzo para obtener la información es más conveniente ir ampliando el número de criterios y eliminar proveedores progresivamente. La búsqueda de proveedores potenciales estuvo basada principalmente en información interna a la empresa, estimándose más conveniente complementarla con información secundaria de directorios o asociaciones como lo hizo la empresa del caso 1.

Los proveedores se seleccionan bajo un principio de satisfacción, el cual se manifiesta terminando la búsqueda cuando existe cierto nivel de complacencia del grupo decisor, lo que lleva a elegir demasiado pronto a un proveedor pasando por alto otras alternativas que pudieran ser mejores. Se identifica la necesidad de proponer metodologías para asegurar la suficiencia de la búsqueda, o bien identificar cuando es necesario ampliarla.

La evaluación de proveedores se hace principalmente siguiendo un procedimiento basado en juicios a pesar de que existen herramientas disponibles (De Boer, 2001) para hacer más objetivo el proceso. El costo sigue siendo un criterio con un peso importante en la decisión, pero no es ya el determinante en la selección de una 3PL, otros criterios como servicio a clientes, eficiencia logística y flexibilidad también se consideran. Los criterios de relación (capacidad de la 3PL para establecer una relación 
de asociación con el comprador) no tienen todavía un reconocimiento a pesar de que en la literatura hay varios estudios que demuestran su importante influencia en los resultados de la tercerización (e.g. Knemeyer and Murphy, 2004). La inclusión de este grupo de criterios presenta el reto de su medición a priori.

Los contratos son el mecanismo básico para la administración de la relación y el riesgo asociado a la cesión del control de la actividad logística. Precisar estos contratos (incluyendo costo del servicio) cuando se tercerizan actividades logísticas complejas conlleva un proceso de negociación y definición de medidas de desempeño conjuntas. Se identifica la necesidad de una investigación más extensa encaminada a entender cuáles son las acciones administrativas, los procesos de intercambio más efectivos y los factores del ambiente de negocios que influyen en el establecimiento de asociaciones de tercerización efectivas y duraderas (Kern y Willcocks, 2002).

Dado que los cuatro casos involucran a grandes empresas multinacionales del sector manufacturero, los resultados de este estudio no son necesariamente representativos de las prácticas de tercerización en México; sin embargo, si estas compañías que son líderes en su sector no han formalizado su proceso de tercerización, es de esperarse que compañías con menos recursos tengan prácticas más rudimentarias. Por lo tanto, con base en la literatura revisada y los casos analizados, se revela un gran potencial para la aplicación y desarrollo de métodos para apoyar la toma de decisiones en tercerización; ésta es un área de oportunidad para establecer vínculos entre la academia y las empresas que consideramos debe ser explorada en nuestro medio.

\section{Referencias}

AFRICK, J. M. y C. S. Calkins, "Does asset ownership mean better service?", Transportation and Distribution, 35 (5): 49-61, 1994.

ANDERSON, E., Chu, W. y B. Waiz, "Industrial purchasing: an empirical explanation of the buyclass framework", Journal of Marketing, 51: 71-86, 1987.

ANDERSSON, D. y A. Norrman, "Procurement of logistics services: a minutes work or a multi-year project?", European Journal of Purchasing \& Supply Management, 14: 3-14, 2002. 
ARROYO LÓPEZ, P. E., J. Gaytán Iniestra y A. Pérez Jiménez, "Manos expertas en la cadena: Tendencias" Énfasis Logística, Año 5, No. 52: 50-64, 2004.

ARROYO, P. E., M. Trujillo y J. Gaytán, "Customer segmentation based on logistics costs", en INFORMS Annual Meeting 2003, Atlanta, GA., EUA, 2003.

BARBAROSOGLU, G. y T. Yazgac, "An application of the analytic hierarchy process to the supplier selection problem", Production and Inventory Management, 38 (1): 14-21, 1997.

DE BOER, L., L. Van der Wagen y J. Telgen, "Outranking Methods in Support of Supplier Selection", European Journal of Purchasing \& Supply Management, 4: 109-118, 1998.

— E. Labro y P. Morlacchi, "A Review of Methods Supporting Supplier Selection", European Journal of Purchasing \& Supply Management, 7: 7589, 2001.

, Van der Wagen, L., Gaytán, J. y Arroyo, P., "Outsourcing of Logistics Activities: Development of a Prescriptive Model", en Proceedings of the 13th Annual IPSERA Conference, Catania, Italia, 2004.

, A bounded rationality model of supplier selection, Working paper, Norwegian University of Science and Technology, Department of Industrial Economics and Technology Management, Trondheim, Norway, 2004.

BOYSON, S., T. Corsi y E. Rabinovich, "Managing Effective Third Party Logistics Relationships: What Does it Take?", Journal of Business Logistics, 20 (1): 73-100, 1999.

BRADYLEY, P., "Third parties gain slow, cautious buyer support", Purchasing, May 18: 51-52, 1995.

CHOY, K. L. y W. B. Lee, "A generis tool for the selection and management of supliré relationships in an outsourced manufacturing environment: the case application of case based reasoning", Logistics Information Management, 15 (4): 235-253, 2002. 
DAUGHERTY, P.J. y P. H. Pittman, "Utilization of time-based strategies: creating distribution flexibility/responsiveness", International Journal of Operations \& Production Management, 15 (2): 54-60, 1995.

GAYTÁN, J., P. Arroyo y L. De Boer, Interactive methods for supplier selection. A public sector application", Procedings of the 14th. IPSERA Conference 2005, in Calvi, R. Merminod, N. editors, pp. 579-589, Archamps, France, 2005.

GAYTÁN, J., P. Arroyo y J. A. de León, Selección de proveedores utilizando técnicas de superación: un caso de estudio, XXXIV Congreso de Investigación y Extensión del Sistema Tecnológico de Monterrey, Monterrey NL., 2004.

VAN HOEK, R. I., "The contribution of performance measurement to the expansion of third party logistics alliances in the supply chain", International Journal of Operations \& Production, 21 (1/2): 15-22, 2001.

INSINGA, R. C. y M. J. Werle, "Linking outsourcing to business strategy", Academy of Management Executive, 14 (4): 58-70, 2000.

KANNAN, V. R. y K. Choon Tan, "Supplier selection and assessment: Their impact on business performance", Journal of Supply Chain Management, 38 (4): 11-20, 2002.

KERN, T. y L. Willcocks, "Exploring relationships in information technology outsourcing: The interaction approach", European Journal of Information Systems, 11: 3-19, 2002.

KNEMEYER, A. M. y P. R. Murphy, "Evaluating the performance of third-party logistics arrangements: A relationship marketing perspective", Journal of Supply Chain Management, 40 (1): 35-51, 2004.

LIEB, R. C. y H. L. Randall, "A Comparison of the Use of Third-party Logistics Services by Large American Manufacturers, 1991, 1994 and 1995", Journal of Business Logistics, 17 (1): 305-320, 1996. 
LONSDALE, C., "Effectively managing vertical supply relationships: a risk management model for outsourcing", Supply Chain Management: An International Journal, 4(4): 176-183, 1999.

MCIVOR, R., "A practical framework for understanding the outsourcing process", Supply Chain Management: An International Journal, 5: 22-36, 2000.

MOORE, K. R., "Trust and relationship commitment in logistics alliances: A buyer perspective", International Journal of Purchasing and Materials Management, 34 (1): 24-37, 1998.

TALLURI, S., "A buyer-seller game model for selection and negotiation or purchasing bids", European Journal of Operational Research, 143: 171-180, 2002.

TRANSPORTE SIGLO XXI, "Calculan costo logístico de México del 15\%.", noviembre: C-E, (2003).

WEBER, C. A., "A data envelopment analysis to approach to measuring vendor performance", Supply Chain Management, 1(1): 28-39, 1996.

WEBSTER, F. E. Jr. y Wind, Y., "A general model for understanding organizational buying behavior", Journal of Marketing, 36: 12-19, 1972.

YIN, R. K. (2003), Case Study Research: Design and Methods, $2^{\text {nd }}$ ed, Sage Publications, Newbury Park. $(\mathbb{A}$ 